\title{
Egresados de la Escuela Nacional de Enfermería y Obstetricia (UNAM): calidad de la formación (2003-2008).
}

\section{UNAM “ S National School of Nursing and Obstetrics (ENEO) Graduates: Formation Quality. (2003-2008).}

Pérez-Cabrera Iñiga,• Müggenburg-Rodríguez, María Cristina,•• Ortiz-Acevedo, Pablo...•

Resumen

Introducción: La evaluación del proceso enseñanza-aprendizaje ofrece elementos para proponer cambios en la formación de enfermeras. La ENEO anualmente estudia el desempeño profesional de sus egresados a tres años de la terminación de su carrea, para retroalimentar su formación. Objetivo: Describir la forma como los egresados visualizan la calidad de la formación recibida durante su aprendizaje formal. Metodologia: Estudio descriptivo de seis generaciones, encuestadas a los tres años de la terminación de su carrera, desde 2003 hasta 2008. Muestra: 360 exalumnos. Recolección: encuesta auto administrada, instrumento con validez de contenido, variables relacionadas con proceso formativo, aplicando estadística descriptiva e inferencial, específicamente el biplot. Desde el punto de vista ético se considera con riesgo mínimo. Resultados: La calidad de la formación teórica fue considerada superior a la calidad de la formación práctica. La congruencia entre la formación recibida y la práctica desempeñada fue evaluada prioritariamente como regular. Las actividades con mejor preparación fueron: cuidados complejos y cuidados sencillos para la resolución de problemas, cuidados para la resolución de necesidades fisiológicas y apoyo integral. El principal eje biplot, en lo cuadrantes valorados como alto y muy alto por su aplicación para la comprensión y cuidado del

Palabras

Clave:

Egresados,

Calidad de formación.

- Candidata a Doctor, profesor de carrera asociado C de tiempo completo de la UNAM.

-. Maestra en Salud Pública, profesor de carrera titular A de tiempo completo de la UNAM.

...Lic. En Enfermería especialista en Rehabilitación, alumno del Programa de Maestría en Enfermería de la UNAM.

RECIBIDO 14 DE MAYO 2010

ENVIADO19 MAYO 2010

ACEPTADO 6 JUNIO2010 


\section{Resumen}

paciente, conjunta a las asignaturas: Proceso salud Enfermedad del Adolescente, Adulto y Anciano (AAA) I y II, Enfermería del AAA II y Enfermería del Niño. Ambos cuadrantes muestran además materias básicas. En el cuadrante de baja aplicación están las metodológicas, administrativas, y de apoyo psicológico y biológico. Las de muy baja aplicación pertenecen al área de las humanidades. La confiabilidad del biplot es de 96.5\%. Discusión y Conclusiones: Las actividades del cuidado de enfermería con mejor preparación son congruentes con asignaturas de mayor aplicación para la comprensión y cuidado del paciente, pero se identifica insuficiente vinculación con asignaturas psicobiológicas y humanísticas.

\section{AbStRact}

Introduction: Assessing the teaching-learning process allows suggest changes in the nursing formation process. In order to feedback these issues, the ENEO assesses its graduate's professional performance every year. Objective: Describe how the ENEO's graduates perceive the quality of their nursing formation. Methodology: Descriptive study of generations surveyed 3 years after their graduation date (2003 to 2008). Sample: 360 ex alumni. Data collection: self-administered survey using a content-valid instrument. The information was analyzed using descriptive and inferential statistics including biplot procedures. The study is considered ethically riskless. Results: The theoretical formation was considered superior in comparison to the practical formation. The match between the formation received and the practice performed was assessed as rather regular. The best perceived activities were problem handling, physiological- needs solutions, and integral support. The subject: teen-adult elder health-illness process I and II, teen-adult-elder nursing II, and child nursing, were assessed within the highest application biplot quadrant. Methodological, administrative psychological and biological support subjects were assessed within the low application quadrant. Humanities subject were assessed within the lowest application quadrant, the overall biplot confidence was 96.5\%. Discussion and conclusions: Nursing activities directly related to care were reported as being more congruent with the patient-care, than the psychological and humanistic subjects.

\section{INTRODUCCIÓN}

Uno de los propósitos fundamentales de la Universidad Nacional Autónoma de México es formar profesionales competentes, dotados de sentido social y conciencia nacional para que, vinculados a las necesidades del país, participen con una perspectiva crítica en la promoción de los cambios y transformaciones requeridos por la sociedad.
En el caso de Enfermería cuyo objeto de estudio es el cuidado a la salud de la persona sana o enferma en su dimensión integral, se busca que el licenciado en enfermería y obstetricia desarrolle habilidades intelectuales como el análisis, la síntesis, el pensamiento crítico, la capacidad de resolver y plantear nuevos problemas, la búsqueda y manejo de información a través de diferentes medios, para la toma de decisiones fundamentadas en la planeación del cuidado para el dominio de los procedimientos y tecnologías básicas del campo de la enfermería transferible a diversas situaciones con el fin de alcanzar actitudes sustentadas en la comprensión y respeto por el otro. ${ }^{1}$ 
La Escuela Nacional de Enfermería y Obstetricia (ENEO) en su quehacer institucional está estrechamente ligada a las necesidades de la sociedad, lo cual exige la creación e implementación de planes y programas de estudio pertinentes y flexibles que ofrezcan una fuerte carga humanística y científica en su contenido académico profesional, que se vea plasmado en el curriculum, considerado éste como la especificación de las instituciones educativas y se refiere al conjunto de criterios, planes de estudio, programas, metodologías y procesos que contribuyen a la formación integral del aprendiz, así como los recursos humanos académicos y físicos para poner en práctica las políticas y llevar a cabo el proyecto educativo institucional. ${ }^{2}$

El Plan de Estudio es considerado como parte central de la estructura curricular que vincula los saberes con el modelo educativo y los paradigmas propios de la disciplina y contiene la fundamentación social y educativa para formar recursos humanos calificados en una disciplina en el cual se integran entre otros elementos: propósitos, objetivos, perfil del egresado y programas de las asignaturas con sus contenidos, métodos, experiencias de enseñanza aprendizaje y formas de evaluación, así como los medios y recursos que serán empleados en el proceso educativo. ${ }^{1}$

El plan de estudios responde a una organización por áreas de conocimiento: Enfermería y Salud en México, Proceso Salud-Enfermedad en las Diferentes Etapas Evolutivas y Proceso Salud-Enfermedad en la Reproducción Humana.

El área Enfermería y Salud en México se compone de un conjun- to de asignaturas de las disciplinas fundamentales, las cuales proporcionan los conocimientos básicos del área de la salud en general y de la enfermería en particular. El dominio de los aprendizajes de estas asignaturas permite cubrir el prerrequisito para estudios más especializados y proporcionan los elementos teórico-metodológicos y técnicos básicos para comprender el campo de la enfermería.

Las áreas Proceso Salud-Enfermedad en las Diferentes Etapas Evolutivas y Proceso Salud-Enfermedad en la Reproducción Humana, incluyen las asignaturas que proporcionan los conocimientos, habilidades y destrezas para el desempeño de la práctica profesional.

Todo proceso de formación educativa requiere evaluación periódica que posibilite la verificación del grado en que se han alcanzado los objetivos generales propuestos en el plan de estudio, ésta puede estar orientada hacia la congruencia interna o hacia la congruencia externa, en el caso presente corresponde a la segunda que se refiere a la evaluación que genera información sobre los resultados de la aplicación de un programa, resultados derivados de los aprendizajes obtenidos a partir de la etapa formal de educación de los alumnos que egresan de la ENEO-UNAM, con el fin de identificar los factores y problemas que pueden alterar este proceso, la actividad educativa ofrece elementos para calificar la calidad de la educación y argumentar la implementación de correcciones y mejoras en la formación de recursos humanos. ${ }^{3}$

El seguimiento de egresados de la Licenciatura en Enfermería y Obstetricia de la ENEO ha sido una preocupación constante para la escuela desde hace mucho tiempo, sin embargo a partir de 1991 se estableció un seguimiento sistemático creando un proyecto de carácter institucional que ofrece productos anuales correspondientes a las generaciones de egreso ${ }^{4}$ el cual ha considerado en lo general los propósitos de la ANUIES, asociación educativa de la educación superior que ha recomendado: comparar los resultados obtenidos en el proceso educativo llevado a cabo por las instituciones de educación superior y las necesidades y exigencias del campo profesional; conocer la coherencia que existe entre los perfiles terminales, los objetivos de los planes y programas y los requerimientos formativos actuales de las distintas prácticas profesionales; conocer las nuevas exigencias que plantea el ejercicio profesional a los egresados como consecuencia de las transformaciones económicas, sociales y tecnológicas para su incorporación oportuna en los planes y programas de estudio; conocer el grado de satisfacción de los egresados respecto a la calidad de la formación recibida; contar con información de las tasas de empleo en los distintos sectores del mercado laboral para afinar las políticas de de formación; contribuir a mejorar la imagen de las instituciones en su área de influencia mediante la difusión de la información sobre el desempeño de sus egresados a fin de fortalecer la confianza de la sociedad en las instituciones educativas. ${ }^{5}$

En cuanto a los hallazgos obtenidos, se han identificado algunas respuestas de éxito o fracaso del proceso formativo de los egresados de la ENEO que nos proporcionan elementos valiosos para el 
trabajo académico cotidiano, como el predominio de la calidad de la formación teórica sobre la formación práctica, la demanda de mayor congruencia entre formación recibida y práctica desempeñada en el mercado laboral, el reconocimiento de una formación educativa orientada hacia el aprendizaje de actividades esenciales de la disciplina como cuidados profesionales técnicos, genéricos asistenciales y humanísticos; ${ }^{6,7,8}$ estos hallazgos son congruentes con otros estudiados realizados dentro de la UNAM y en otros centros educativos. $., 10,11$

En este reporte se describe la manera en la que los egresados de la ENEO en los años 2000, 2001, 2002, 2003, 2004 y 2005 visualizan la calidad de la formación recibida durante su etapa de aprendizaje formal.

\section{METODOLOGÍA.}

Estudio observacional descriptivo de seis generaciones de egresados de la ENEO, de 2000 a 2005, quienes se estudian a los tres años de la terminación de su carrera, de 2003 a 2008. Con una muestra aleatoria de 360 exalumnos, obtenida a través de un muestreo sistemático, apoyado en las listas de los egresados que cumplen con el 100\% de los créditos del plan de estudios.

Las variables seleccionadas para este análisis se dirigen a: la calidad de la formación teórica y de la formación práctica recibida durante su proceso formal educativo, a la congruencia experimentada entre la formación recibida y la práctica desempeñada en el ámbito profesional, valoradas con escalas tipo Likert que clasifican desde un máximo hasta un valor nulo.

También se exploran las actividades para las cuales en su opinión

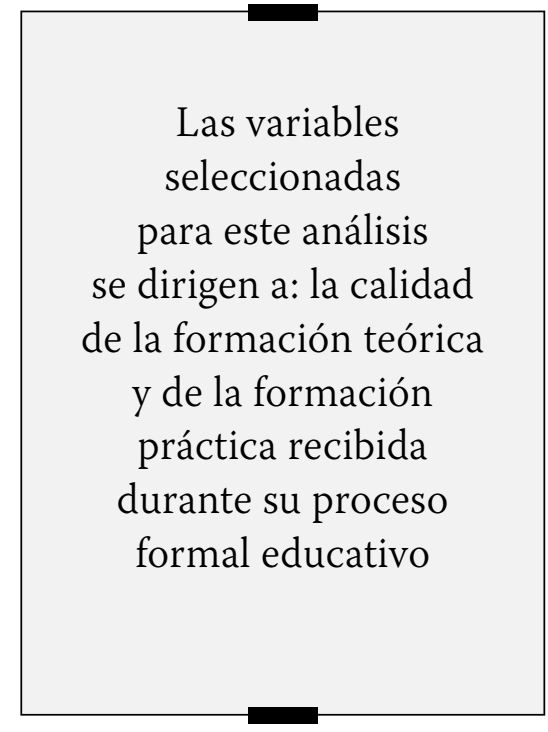

reciben mejor preparación, a partir de un listado que reúne actividades de caracter asistencial, técnico, humanístico, educativo, docente, administrativo e investigativo. Así como, el nivel de aplicación de las asignaturas para la comprensión y cuidado de los pacientes, cuyos parámetros se establecen desde niveles mínimos hasta niveles máximos.

La técnica de recolección de la información propuesta para la consecución de los objetivos se circunscribe a un interrogatorio indirecto (encuesta), apoyado en un instrumento integrado por preguntas, en su mayoría cerradas, que exploran variables relacionadas con: datos generales de los egresados, formación académica y situación laboral; para cumplir con el propósito de este reporte se abordan las variables, citadas en los párrafos anteriores, relacionadas con la formación académica, dejando para otro momento los aspectos laborales.

La estrategia para la aplicación de la encuesta se concreta en lo siguiente: se selecciona una muestra de la población que egresó de la escuela tres años antes de la fecha del seguimiento planeado, es decir exa- lumnos que terminaron en 2000, $2001,2002,2003,2004$ y 2005 . Una vez determinada la muestra se procede a la localización telefónica de los exalumnos seleccionados, considerando su domicilio personal; logrado este primer contacto se le cita en la ENEO o se establece un compromiso para visitarlos en su casa o en su centro de trabajo con el fin de concertar una entrevista para recolectar la información.

Después de la recopilación de los datos se procede a su captura y concentración con el apoyo del programa SPSS versión 17, para aplicar pruebas estadísticas que permitan la descripción, el análisis y la interpretación. Con el fin de apreciar de una manera fehaciente las relaciones entre dos conjuntos de variables y condensar simultáneamente en una sola grafica información que, de otra manera, debería ser analizada mediante tablas sucesivas, se utilizo el biplot que permite evaluar visualmente la proporción de variabilidad de cada una de las variables que es explicada por variables predictoras, ${ }^{14}$ En el caso presente, la prueba multivariada del biplot retrata las diferencias entre los conglomerados o clusters de materias obligatorias del plan de estudios de la licenciatura en Enfermería y Obstetricia de la UNAM y el atributo asignado por los exalumnos de las generaciones encuestadas, como respuesta al cuestionamiento: cuál es el nivel de aplicación de las asignaturas del Plan de Estudios (1991) para la comprensión y el cuidado brindado a los pacientes durante los tres años de experiencia profesional, la opción para los atributos fue: muy bajo (1), bajo (2), medio (3), alto (4) y muy alto

(5). Esta prueba arroja un gráfico 
que muestra las relaciones entre las materias y los atributos asignados por los exalumnos, mismo que será mostrado y analizado en el capítulo correspondiente.

Desde el punto de vista ético, se establecen los mecanismos necesarios para proteger la integridad de los participantes, guardando la confidencialidad de los datos recolectados.

\section{Resultados}

La población estudiada corresponde a una muestra de 360 alumnos que egresaron con el $100 \%$ de sus créditos desde 2000 hasta 2005, con una edad promedio de 28 años y predominio del género femenino. Durante su etapa de formación académica mantuvieron un promedio de calificación que osciló entre 8.3 y 8.7, sin embargo alrededor del $45 \%$ manifestó haber reprobado alguna materia durante su trayectoria escolar, entre las que destacan: anatomía y fisiología, y farmacología.

Del 69\% al 91\% de los egresados opinaron que a la calidad de la formación teórica que reciben corresponde al grado de "mucha" y del 6\% al 29\% la calificaron como "regular", registrando una imagen en espejo entre ambas, la apreciación de "poca" y "nula", es muy baja. Respecto a la calidad de la formación práctica, guardó una tendencia similar pero con valores inferiores, la calificación de "mucha" oscila entre el $51 \%$ y el $70 \%$, mientras que la de "regular" oscila de 29\% a 41\% (Gráfica No.1).

La congruencia experimentada entre la formación recibida y la práctica desempeñada en el mercado laboral fue calificada como "regular", desde el 37\% hasta el $64 \%$, mientras que la calificación de "mucha", osciló del 26\% al 49\%. La apreciación de "poca" y "nula" es muy baja (Gráfica No.2).

Los egresados consideraron que las actividades para las cuales reciben mejor preparación son: los cuidados complejos tendientes a la resolución de alteraciones patológicas en el enfermo, seguidos de cuidados sencillos, cuidados enfocados a las necesidades fisiológicas y cuidados enfocados al apoyo integral del paciente y su familia (Gráfica No.3).

De acuerdo con el análisis realizado a través de la gráfica del biplot se puede apreciar la aglutinación de 5 clusters o conglomerados, que corresponden a los siguientes grupos de materias: a) Obstetricia I y Obstetricia II; b) Anatomía y Fisiologíar y II, Farmacología, Enfermería del niño, Proceso Salud Enfermedad en el Adolescente, Adulto y Anciano I y II, Enfermería en el Adolescente, Adulto y Anciano I I y II; c) Fundamentos de Enfermería I y II y Proceso Salud Enfermedad en el niño; d) Nutrición Básica y Aplicada, Metodología de la Investigación, Ecología y Salud, Desarrollo de la Personalidad Infantil, Desarrollo de la Personalidad del Adolescente, Adulto y Anciano, Administración General, Ética Profesional y Legislación, Administración de los Servicios de Enfermería y Docencia en Enfermería; e) Historia de la Enfermería, atención a la Salud en México, Socioantropología, didáctica de la Educación para la Salud y Psicología General.

La organización de los 5 conglomerados también permite observar que la relación entre unos y otros no es al azar, como puede identificarse en la gráfica No. 4 , ya que la cercanía entre las asig- naturas del conglomerado a) y las del b), guardan una relación entre ellas, por ejemplo las Obstetricias están cercanas a la Anatomía y a la Farmacología, están en la parte superior del mismo cuadrante; así como las asignaturas del grupo d), como es el caso de la Metodología de Investigación tiene relación con Didáctica de la Educación para la Salud, ya que ambas aún cuando se sitúan en cuadrantes diferentes se encuentran cercanas al eje 0.0 de la $\mathrm{x}$.

Además de las observaciones ya señaladas, es importante mencionar que los conglomerados localizados en los cuadrantes localizados a la derecha del eje 0.0 de la y, corresponden a las asignaturas valoradas por los alumnos como materias que tienen un nivel de aplicación muy alto y alto para la comprensión y cuidado del paciente brindado durante los tres años de experiencia como profesionales de la enfermería, así como las grupos localizados hacia la izquierda del eje 0.0 de la y, corresponden a las valoradas como medio y bajo.

Se observa que el principal eje biplot conjunta a las siguientes asignaturas: Proceso Salud Enfermedad del Adolescente, Adulto y Anciano I y II, Enfermería del Adolescente, Adulto y Anciano II y Enfermería del Niño. Pero en ambos cuadrantes se observan materias básicas de apoyo como: Anatomía, Fisiología y Farmacología, así como asignaturas de Enfermería; Fundamentos de Enfermería I y II, Enfermería del Adolescente, adulto y Anciano I; y otras como Proceso Salud Enfermedad del Niño y Obstetricia I y II.

En el cuadrante que conjunta la categoría de materias valoradas 
como de baja aplicación se encuentran: Metodología de la Investigación, Administración de los Servicios de Enfermería, Administración General, Desarrollo de la Personalidad del Adolescente, Adulto y Anciano, Desarrollo de la Personalidad Infantil, Ecología y Salud y Nutrición Básica y Aplicada.

Por último, el cuadrante que corresponde a la categoría de muy baja aplicación, se reúnen las siguientes asignaturas: Psicología General, Didáctica de la Educación para la Salud, Socioantropología e Historia de la Enfermería.

\section{Discusión}

El perfil de los exalumnos de las seis generaciones es similar al de egresados de años anteriores, tanto en aspectos socio demográficos como escolares: los egresados de de las diversas generaciones siempre han considerado que su formación teórica es de más calidad que su formación práctica; ${ }^{12}$ para lo cual podría haber diversas lecturas, como la falta de experiencia profesional de algunos docentes con sus respectiva consecuencia, el uso de campos inadecuados durante las prácticas escolares, o expectativas muy ambiciosas de los estudiantes. Dichas opiniones están muy relacionadas con la congruencia experimentada entre la formación recibida y la práctica desempeñada en el mercado laboral que la mayoría calificó con el nivel de regular. ${ }^{13}$ De igual manera los aprendizajes identificados por ellos como más importantes se mantienen en lo general como en estudios previos ${ }^{14} y$ se centran en las actividades inherentes a la profesión.

La agrupación de materias como Anatomía, Fisiología y Far- macología de carácter biologicista con la Obstetricia permite identificar la necesidad que atribuyen los exalumnos al conocimiento de ciencias básicas para el desempeño de la Obstetricia, lo que confirma la ubicación de esta materia en los últimos semestres del Plan de Estudios. $^{15}$

La relación entre la Didáctica de la Educación y la Metodología de la Investigación será un punto interesante por estudiar, ya que los estudios de investigación orientados a la enseñanza, ofrecen elementos para el desarrollo de prácticas educativas con su consecuente reducción de riesgos para la salud; ${ }^{16} \sin$ embargo ambas asignaturas fueron clasificadas como de muy baja y baja aplicación, situación que tiene semejanza con los hallazgos de un estudio realizado en la FES Iztacala, pues aún cuando su plan de estudio es modular, se aprecia que los módulos que contienen estos aprendizajes, también fueron calificados por los alumnos como de baja contribución para el logro de su Perfil Profesional. ${ }^{17}$

La relación observada en el principal eje Biplot entre las Enfermerías y Fundamentos de Enfermería con materias básicas (Anatomía, Farmacología Proceso Salud Enfermedad), una vez más evidencia el lugar que los alumnos atribuyen a las ciencias biomédicas para la comprensión y aplicación del cuidado al paciente, observación congruente con las actividades que realizan los egresados de otras escuelas, prioritariamente del ámbito asistencial. $^{18}$

Entre las materias consideradas por los exalumnos como de baja o muy baja aplicación se señalan algunas que aún cuando son de apo- yo tienen un lugar esencial para el logro de la identidad profesional como es la Historia, ${ }^{19}$ para la eficiente comunicación enfermerapaciente, como son la Psicología y el Desarrollo de la Personalidad en sus diferentes etapas, así como para la aplicación de los cuidados en el entorno familiar y comunitario como son la Socioantropología y la Didáctica para la Salud.

Estas reflexiones son importantes para los docentes que imparten dichas asignaturas de apoyo, ya que deberán vincular estos aprendizajes de manera explícita para que los alumnos comprendan la importancia que estos tienen en el desempeño de sus prácticas favoreciendo el cuidado holístico.

\section{Conclusiones}

- La formación teórica es mejor calificada que la formación práctica, por lo que esta última debe reforzarse con condiciones idóneas de aprendizaje sustentadas en el ejercicio de la práctica y en la experiencia de los docentes, aspectos que también coadyuvan para la apreciación de la congruencia entre el aprendizaje y el desempeño profesional.

- La identificación de los grupos de materias y la relación de éstas en el contexto del plan de estudios induce a la reflexión de la formación recibida durante su etapa de aprendizaje formal con el fin de buscar estrategias que refuercen los saberes que las profesionales de enfermería necesitan en su práctica cotidiana. 


\section{Enfermería Universitaria}

- La valoración sobre las actividades del cuidado de enfermería para las cuales reciben mejor preparación es coherente con la opinión de las asignaturas que tiene mayor aplicación para la comprensión y cuidado del paciente, sin embargo se identifica una insuficiente vinculación entre asignaturas de carácter psicobiológico y de carácter social, relación que es esencial para la comprensión del cuidado integral.

\section{REFERENCIAS BIBLIOGRAFICAS}

1 ENEO-UNAM. Plan de estudios de

la Licenciatura en Enfermería y Obstetricia. México: ENEO, 2000. p. 38-39

2 Sacristán, Gimeno J. \& Pérez Gómez, A.I. Comprender y transformar la enseñanza. 4a ed. Madrid: Ediciones Morata, 1995 p. 141-156

3 Díaz-Barriga, F. Hernández-Rojas, G. Estrategias docentes para un aprendizaje significativo. Una interpretación constructivista. $2^{\text {a }}$.ed. Mc Graw Hill, México 2007 p.11

4 Müggenburg, C. Seguimiento de egresados de la ENEO. Acontecer Académico. ENEO UNAM. Febrero de 2004. Año27, No. 2 pp.16-17

5 ANUIES: esquema básico para estudios de egresados. México. Biblioteca de la Educación Superior Serie Investigaciones 2003 p.11
6 Müggenburg, C. Pérez Cabrera, I. y Cols. Seguimiento de Egresados de la ENEO UNAM 2004 Metamorfosis en Enfermería Vol.1 No.4 Mayo-Junio 2006 p. 5-10

7 Müggenburg C y Cols. Seguimiento de Egresados de la Licenciatura en Enfermería de la ENEO en 2000 a los tres años (2003) Enfermería Universitaria Vol.3 No.1 EneroAbril 2006 p. 5-13

8 Müggenburg C. Pérez Cabrera I. y Cols. Seguimiento de egresados de la licenciatura de la ENEO-UNAM formación y desempeño laboral (2003-2006) Enfermería Universitaria Vol.5 Año 1 Enero-Marzo 2008 p. 21-29

9 Crespo Knopfler, S., et. al., Percepción de empleadores sobre el licenciado en Enfermería de la Facultad de Estudios Superiores Zaragoza. Revista Enfermería Universitaria. ENEO - UNAM, Vol.6, Año 6, No. 12009.

10 González Velázquez, Susana y cols. Seguimiento de egresados da la Licenciatura de Enfermería. Desarrollo Científico de Enfermería. Vol.17 No.3 Abril, 2009 p.17-19

11 Lopera, C., Los estudios sobre seguimiento de egresados en los procesos de mejora continua de la actividad universitaria, en línea, disponible en: http://www. gradua2.org.mx/docs/monterrey/ Ponencia_U_Catolica_de_Col_ GRADUA2_Mexico.doc, consultado el 20 de febrero de 2010
12 Cárdenas, O. Galindo, P. y VicenteVillardón, J.L. Los métodos Biplot: Evolución y aplicaciones. Análisis de coyuntura (online) junio 2007 Vol.13 No.1 (citado 17 de marzo de 2010), p.279-303 Disponible en www: http://www2.scielo.org.ve/ scielo.php?script=sci_artext\&pid=S131536172007000100013\&Ing=es\&nr $\mathrm{m}=$ iso $>$.ISSN 1315-3617

13 Müggenburg, C. Castañeda, E. Franco, W. Seguimiento de egresados de la ENEO en 1995, a los tres años de su egreso (1998) Enfermeras Vol.36 No.2 2000 p.4-17

14 Frías Osuna, A. Salud Pública y Educación para la Salud. Barcelona: Mason, 2000, p. 339-432

15 García Hernández, P. Factores que determinan el logro del Perfil Académico Profesional de los alumnos de la carrera de Licenciatura en Enfermería de la FES Iztacala. Tesis para obtener el grado de Maestría en Enfermería del Programa de Maestría en Enfermería de la UNAM 2009 p.54

16 Flores, C. Enseñanza de la Historia. Correo del Maestro Núm. 102, noviembre de 2004. Disponible en http://correodelmaestro.com/ anteriores/2004/noviembre/libros102.htm Consultado el 23 de marzo de 2009.

\section{Agradecimientos.}

A la Maestra Reyna Matus Miranda por la revisión del manuscrito. 
Gráfica 1.

Calidad de la formación teórica y práctica recibida por los egresados de la licenciatura de la ENEO-UNAM de 2003 a 2008

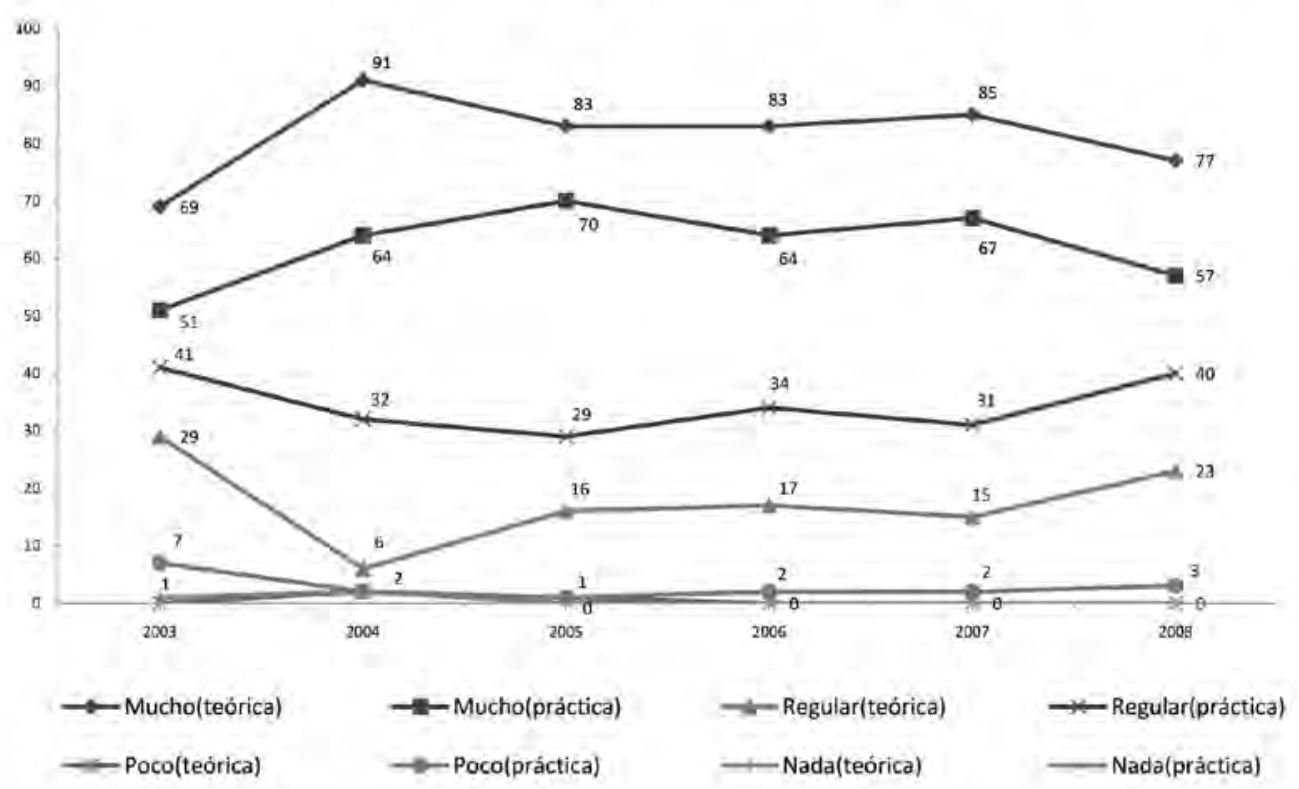

Fuente: Informes del seguimiento de Egresados de la Licenciatura en Enfermeria y Obstétricia de la ENEO UNAM 2003-2008

\section{Gráfica 2.}

Congruencia experimentada por los egresados de la ENEO UNAM entre la formación recibida y el mercado laboral de 2003 a 2008

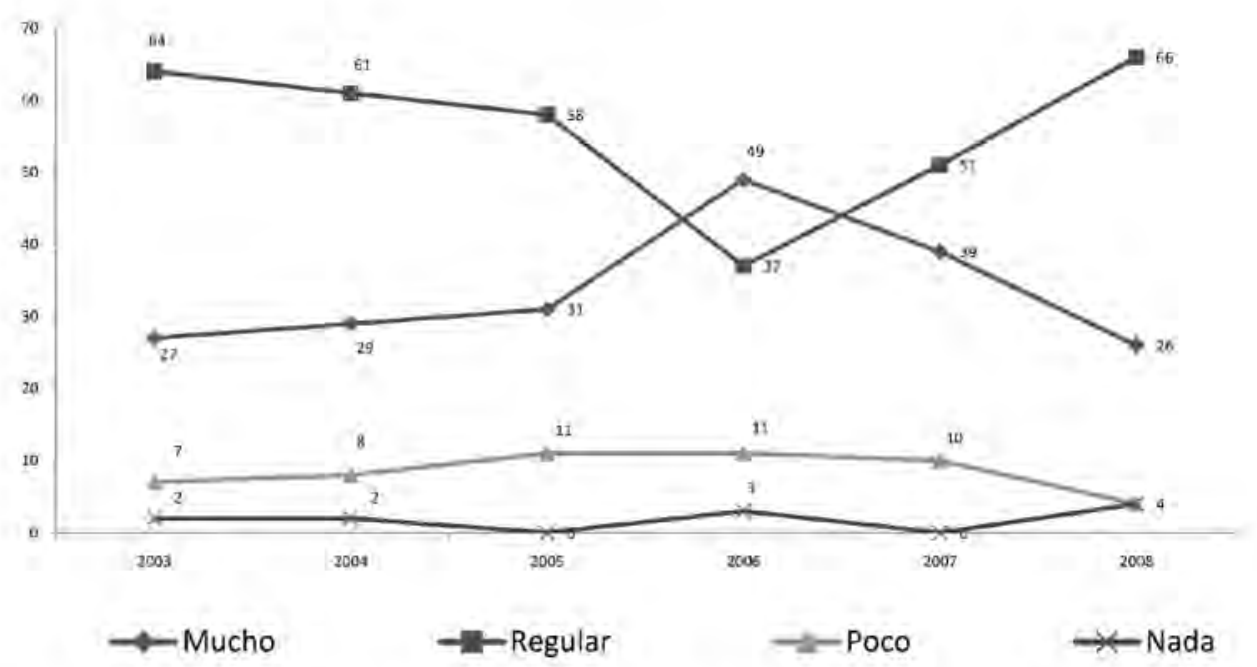

Fuente: informes del Seguimiento de Egresados de la Licenciatura en Enfermería y Obstetricia de la ENEO UNAM $2003 \cdot 2008$ 


\section{Gráfica 3.}

Actividades con mayor preparación según opinión de los egresados de la Licenciatura de la ENEO UNAM de 2003-2008

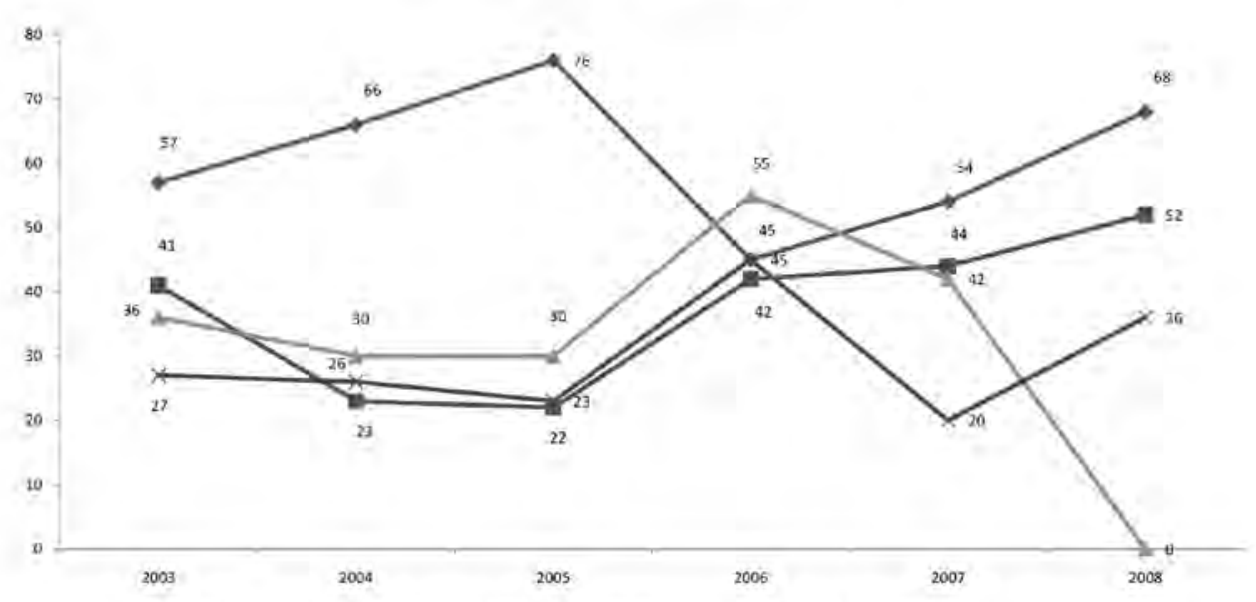

- Cuidados de Enfermeria complejos tendientes a la resolución dẹ alteraciones patológicas en el enfermo.

$\rightarrow$ - Cuidados de Enfermería sencillos tendientes a la resolución de alteraciones patólogicas reales o potenciales del enfermo en hóspitaización

_Culdados de Enfermería enfocados a la rasolución de necesidades fisiológicas del enfermo en hospitaies.

$\rightarrow$ - Culdados de Enfermeria enfocados at apovo intogral del paciente y su familia:

Fuente: Informes del Seguimiento de Egresados de la Licenciatura en Enfermeria y Obstetricia de la ENEO UNAM 2003-2008

\section{Gráfica 4.}

Nivel de aplicación de las asignaturas del Plan de Estudios que permiten la comprensión y cuidado de los pacientes. Egresados ENEO-UNAM 2003-2008

1 Historia de la Ent. de Mexioo 2 Atención a la salud en México

3 Socioantropologia

4 Metodologia de la investigación

5 Anatomia y Fisiologia I

6 Anatomía y Fisiologia II

7 Ecologia y Salud

a Psicologia General

9 Fundamentos de Entermeria I

10 Farmacología

11 Nutrición Básica y Aplicada

12 Fundamentos de Ent . II

13 PSE en el niño

14 Enfermeria del niño

15 Desarrallo de la Personalidad Intantit

16 Didactica de la Educ. para la salud

17 PSE en el AAAI

18 Eniemeria en el AAAI

19 Des de la personalidad del AAA

20 Administración Genera

21 PSE en el AAAI

22 Enfermeria en al AAAll

23 Ética Profesional y Legislación

24 Administración de los Servicios de Eni

25 Obstetricia !

26 Docencia en enfemeria

27 Obstetricia II

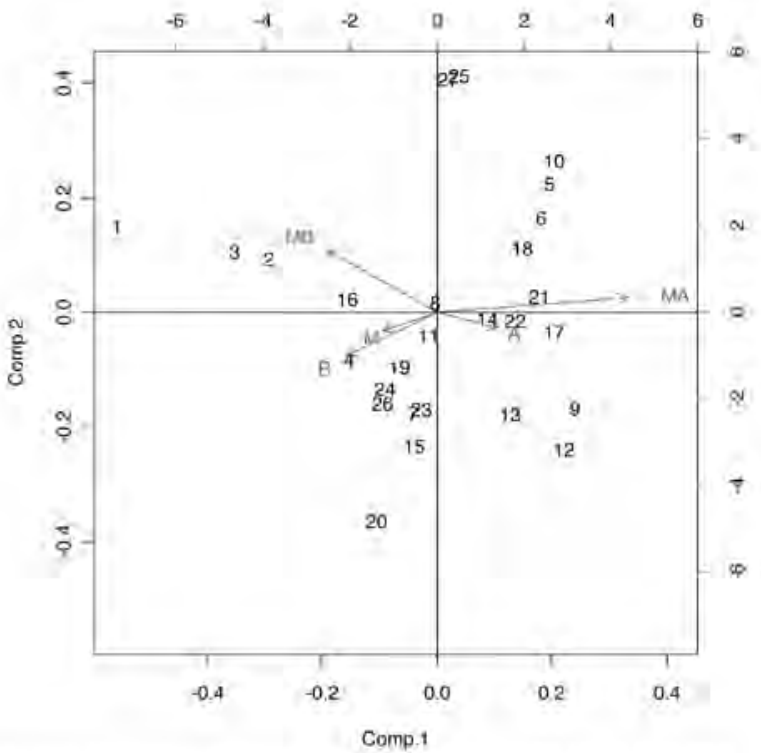

Fuente Iniomes del Seguimiento de Egresados de la Licenciatura en Enfermeria y Obstetricia de la ENEO UNAM 2003-2008 